\title{
Ascenso y hegemonía: pensando a las potencias emergentes desde América del Sur ${ }^{1}$
}

\author{
Rise and hegemony: some observations on emerging powers \\ from a South American perspective
}

LUIS L. SCHENON/*

Rev. Bras. Polít. Int. 55 (1): 31-48 [2012]

\section{Introducción}

En la segunda década del siglo XXI, la percepción del surgimiento de nuevas potencias o estados emergentes comienza a cuestionar profundamente nuestra forma de concebir la política internacional. Se ha formado un amplio consenso en torno a la idea de que el poder en el mundo se encuentra más desconcentrado, especialmente en comparación con las dos décadas posteriores a la guerra fría, lo que estaría dando paso a un mundo más o menos multipolar, a un ascenso del resto (Zakaria 2008), que incluiría a Brasil, pero no necesariamente al resto de Sudamérica.

En este trabajo pretendemos resumir las explicaciones realistas a este cambio en la política internacional a partir del «realismo neoclásico» (Rose 1998) y algunas ideas propias del «realismo de la periferia» (Russell y Tokatlian 2010). Para ello nos enfocaremos en el caso de una potencia emergente en particular: Brasil, e intentaremos desentrañar los determinantes de su ascenso y potencial hegemonía sobre la región.

A continuación, en un primer apartado, analizaremos cómo el debate sobre las potencias emergentes encuadra en un corpus conceptual y teórico realista que aún así presenta limitaciones para explicar los procesos de cambio. En un segundo

\footnotetext{
1 Una versión más extensa de este trabajo fue presentada en el X Congreso Nacional de Ciencia Política, organizado por la Sociedad Argentina de Análisis Político (SAAP) y la Universidad Católica de Córdoba (UCC) en Córdoba, Argentina, del 27 al 30 de julio de 2011, obteniendo el Premio «Relaciones Internacionales» de la SAAP. Agradezco profundamente los comentarios de los asistentes a dicha reunión y muy especialmente los de Roberto Russell, Juan Gabriel Tokatlián, Jorge Battaglino, Fabián Calle, Marcelo Saguier, Federico Merke, Lucas González, Ignacio Labaqui, Santiago Alles, Marcelo Raimundo da Silva y un evaluador anónimo de RBPI.

* Becario doctoral del Consejo Nacional de Investigaciones Científicas y Técnicas (CONICET). Profesor Asistente en la Licenciatura en Relaciones Internacionales de la Universidad Católica Argentina (UCA) y estudiante de la Maestría en Estudios Internacionales de la Universidad Torcuato Di Tella (UTDT) (llschenoni@gmail.com).
} 
apartado intentaremos poner en diálogo la teoría de la estabilidad hegemónica con la teoría del balance de poder, dos tradiciones intelectuales con inferencias importantes en el análisis de este proceso. Luego, en el tercer y cuarto apartados, construiremos un modelo de interacción entre estos dos tipos ideales de orden que constituirá un aporte a una teoría realista del cambio. Por último, intentaremos explicar la historia del ascenso de Brasil en los últimos veinte años y la falta de reacción de la región a partir del caso argentino.

\section{Estado del arte: las potencias emergentes en el realismo}

En las relaciones internacionales, la reflexión sobre la situación relativa de poder de determinados estados frente a sus pares, dejando entre paréntesis otros actores y procesos, ha sido el lugar común de la escuela realista en sus más diversas formulaciones. Siendo así, no debería sorprendernos que la terminología de este debate deba mucho a este enfoque ni que sus referentes se hayan abocado a estudiar este fenómeno con especial interés. La idea de polaridad, largamente debatida por la escuela realista es crucial para comprender la forma que toma este proceso de redistribución del poder, que se concibe a grandes rasgos como el paso de un mundo unipolar a un mundo multipolar en ciernes, o en otros términos: como el tránsito a un nuevo equilibrio con nuevas potencias.

También es interesante la idea de emergente, pues no hace referencia a un proceso de cambio indistinto, sino a las fuerzas naturales que hacen físicamente inevitable el surgimiento de un cuerpo hacia la superficie. Este planteo estructuralista debe su fuerza al principal teórico neorrealista, Kenneth Waltz, quien en la temprana postguerra fría forjaría el concepto más recientemente popularizado de potencias emergentes (Waltz 1993). En aquellos años, mientras algunos nuevos enfoques postulaban la posibilidad de extender en el tiempo la primacía americana a través del mantenimiento del orden institucional vigente o la oportunidad que la situación representaba para moldear el mundo según sus intereses, el neorrealismo se reformuló notablemente para explicar las inconsistencias históricas del balance de poder (Schweller 1998; Wohlforth 1993; Snyder 1991; Friedberg 1988; Zakaria 2000). Estos autores debieron explorar los determinantes domésticos de la política exterior y cómo estas variables informaron distintas respuestas frente a iguales presiones sistémicas, retomando las ideas de realistas clásicos. Igualmente, para estos realistas «neoclásicos» (Rose 1998) como para Waltz, dado que los incentivos del sistema internacional siempre priman, el mundo debía evolucionar necesariamente hacia un escenario donde las principales potencias de segundo orden reestructurarían el sistema internacional de equilibrio de poder.

De esta forma, la problemática de las potencias emergentes se puede identificar fácilmente en el programa realista de las últimas dos décadas y las explicaciones más difundidas se ajustan notablemente a los ideales de conducta y mundos posibles 
de esta teoría. Asimismo, desde un punto de vista normativo, la narrativa realista promueve la regeneración de un mundo según los patrones clásicos, que desestima las últimas dos décadas a modo de un simple paréntesis y favorece el retorno de la historia del balance de poder, encarnada en nuevos actores.

Sin embargo, el realismo ha demostrado una dificultad persistente para explicar el cambio en la política internacional, característica esencial de este y cualquier otro proceso político. Aunque los aportes neoclásicos han logrado otorgar una cierta flexibilidad al neorrealismo, el temor a perder en parsimonia teórica (Walt 2002) ha mantenido a todos los miembros de esta corriente aferrados a una ley: los incentivos sistémicos siempre terminan primando a favor de un equilibrio. A nuestro entender esta proposición contradice una lógica intrínseca del proceso político que pretendemos analizar y es que el ascenso de las potencias emergentes, a la vez que equilibra el sistema internacional, produce grandes desequilibrios - potenciales hegemonias - en los niveles subsistémicos o regionales. A la inversa, resulta evidente que la persistencia de equilibrios subsistémicos supone y permite un escenario de unipolaridad o hegemonía en el nivel sistémico. Esta lógica, ya percibida por Samuel Huntington (1993) explica particularmente bien el caso del ascenso de Brasil en nuestra región ¿̨Por qué habrían de primar los incentivos sistémicos para que Brasil balancee a los Estados Unidos y no aquellos que llevarían a otros estados de la región como la Argentina a balancear a su principal competidor regional?

\section{Una teoría realista del cambio}

Si los estados se comportaran perfectamente de acuerdo a los supuestos de Waltz, en rigor, no habría cambio en la política internacional. ${ }^{2}$ En un equilibrio en condiciones de información perfecta, una mínima modificación en las relaciones de poder provocaría el balance de contrapartes muy sensibles al cambio (Grieco 1993). En la realidad estos cambios en el poder relativo existen, sería ingenuo negarlo ${ }^{3}$, pero para un neorrealista ultramontano, los desbalances se solucionan rápido o terminan indefectiblemente en un conflicto armado. En suma, la teoría del equilibrio de poder en su formulación estructuralista ve el cambio en las capacidades relativas como algo difícil en sí mismo, el cambio de polaridades o estructura como un fenómeno frecuentemente precedido por la guerra, y al cambio de un sistema de equilibrio a uno hegemónico como algo imposible en los términos de la teoría.

Una segunda teoría racionalista y realista con corpus propio que explica muy bien el status quo internacional es la teoría de la estabilidad hegemónica (Gilpin 1981). A la inversa de lo que sucede en el mundo de Waltz, la hegemonía es aquí el único sistema estable posible. El cambio difícilmente se produce por la relación

2 Véase el trabajo de Randall Schweller (1996) sobre los sesgos statuquistas del neorrealismo.

3 Como aclara Wohlforth $(1995,102)$ «Waltz siempre admitió que causas al nivel de la unidad podían retrasar el efecto de los incentivos sistémicos por períodos prolongados». 
asimétrica que el hegemón ostenta e intenta prolongar en el tiempo; de producirse un cambio, este debería ser irrelevante o terminar violentamente en una "guerra hegemónica» que enfrente al hegemón en decadencia con su principal competidor. Como resultado de este conflicto puede prevalecer la potencia dominante, surgir una nueva hegemonía — cambio sistémico — o producirse una redefinición de las unidades políticas constitutivas del sistema — cambio de sistema- La posibilidad del cambio hacia un sistema de equilibrio es, desde esta perspectiva, inconcebible.

Nótese que ambas corrientes, así planteadas, son mutuamente excluyentes. La hegemonía no puede existir jamás para los teóricos del balance del poder y el equilibrio jamás podría existir para los teóricos de la estabilidad hegemónica. Para ambas, cualquier intento de acercamiento al orden opuesto llevaría axiomáticamente a una guerra que reestablecería sus respectivos mundos ideales.

En suma, estos enfoques: (i) se basan en supuestos realistas y racionalistas; (ii) son estructuralistas, teorizan la estabilidad pero no el cambio; (iii) uno explica un sistema jerárquico o vertical y otro un sistema anárquico u horizontal; y (iv) se presentan como opuestos, no habiendo intentos de integración o diálogo teórico.

Desde nuestro punto de vista, el camino tomado por los realistas neoclásicos no solo puede ayudarnos a explicar las inconsistencias en el balance de poder sino que la introducción de variables domésticas al análisis de la politica internacional permite concebir el cambio de sistemas de equilibrio a sistemas hegemónicos y viceversa. Esta visión propone, en síntesis, un programa realista ampliado e integrado.

Así como existen transiciones entre escenarios de diferentes polaridades o cambios estructurales —en el marco del equilibrio de poder — o cambios sistémicos, es decir, de hegemón — en la teoría de la estabilidad hegemónica — podrían existir cambios de situaciones de equilibrio a escenarios hegemónicos en un mismo nivel. En adelante llamaremos a este fenómeno cambio de orden.

Es sensato pensar que antes de establecido el escenario hegemónico que caracterizó a la pax britanicca, el escenario continental europeo era semejante al de un equilibrio de poder. Trabajos célebres como Después de la Hegemonía de Robert Keohane (1984) reconocieron implícitamente la posibilidad de que una situación de hegemonía se convirtiese en una situación de equilibrio como se presumió, por ejemplo, en relación al ascenso de Alemania y Japón en los años setenta. Sin embargo, aunque el cambio del equilibrio a la hegemonía - y viceversa- era lógicamente posible, no fue teorizado.

Asimismo, si lográsemos integrar estas dos corrientes realistas al análisis de la política internacional en un determinado momento histórico podríamos ver la interacción de escenarios de equilibrio y hegemónicos en distintos niveles como el internacional y el regional (Mearsheimer 2001). Podríamos decir que las grandes potencias que configuraron un equilibrio gozaron generalmente de algún grado de hegemonía en sus regiones próximas. Algunos casos históricos y prospectivos de cambio entre equilibrios y hegemonías y su interacción en diferentes niveles del sistema puede observarse en el Cuadro 1. 
Cuadro 1. Interacción y cambio entre los conceptos de equilibrio y hegemonía.

\begin{tabular}{|l|l|l|l|}
\hline & Guerra Fría & Postguerra Fría & Escenarios Futuros \\
\hline Plano internacional & $\begin{array}{l}\text { Bipolaridad: } \\
\text { Estados Unidos } \\
\text { Unión Soviética }\end{array}$ & $\begin{array}{l}\text { Unipolaridad: } \\
\text { Estados Unidos }\end{array}$ & $\begin{array}{l}\text { Multipolaridad: } \\
\text { Estados Unidos - } \\
\text { Potencias emergentes }\end{array}$ \\
\hline Plano hemisférico & $\begin{array}{l}\text { Hegemonía: } \\
\text { Estados Unidos }\end{array}$ & $\begin{array}{l}\text { Hegemonía/ } \\
\text { Unipolaridad: } \\
\text { Estados Unidos }\end{array}$ & $\begin{array}{l}\text { Bipolaridad: } \\
\text { Estados Unidos - Brasil }\end{array}$ \\
\hline Plano regional & $\begin{array}{l}\text { Bipolaridad: } \\
\text { Argentina - Brasil }\end{array}$ & $\begin{array}{l}\text { Unipolaridad: } \\
\text { Brasil }\end{array}$ & $\begin{array}{l}\text { Hegemonía: } \\
\text { Brasil }\end{array}$ \\
\hline
\end{tabular}

Si consideramos el cambio de orden como una posibilidad, sería lógico pensar que dicho cambio se produce únicamente tras momentos de grave tensión y conflicto. Sin embargo, en la historia reciente, el paso del «equilibrio del terror» a la unipolaridad ${ }^{4}$ con el fin de la guerra fría o la ruptura del equilibrio subregional y el ascenso de Brasil vis à vis Argentina (Martin 2006, 55), representan cambios asimilables a nuestro tipo ideal de cambio de orden que no han implicado un conflicto. El primer ejemplo inspira gran parte de los trabajos de la corriente neoclásica, mientras que el segundo nos servirá para contrastar empíricamente los postulados que desarrollaremos a continuación. ${ }^{5}$

\section{Una explicación realista neoclásica del cambio: canales de comunicación y socialización}

Los autores neoclásicos han propuesto explicaciones para todo tipo de conductas presumiblemente irracionales o «anómalas» bajo los preceptos del equilibrio de poder y que, adherimos nosotros, podrían llevar a un cambio de orden. Desde la expansión progresiva de una potencia (Zakaria 2000) hasta la sobreexpansión (Snyder 1991), pasando por la adaptación de un poder a su

\footnotetext{
4 En este trabajo consideramos a la unipolaridad como un tipo disminuido de hegemonía o un grado menos en un continuo de descentralización-centralización del poder en un sistema. De acuerdo a grados de centralización crecientes un sistema puede ser no polar, multipolar, tripolar, bipolar, unipolar y hegemónico, hasta llegar a la idea de estado-universal o imperio-universal (Wilkinson 1999, 142). Con esto no pretendemos decir que exista únicamente una diferencia de grado entre la unipolaridad y la hegemonía; al contrario, estas son las dos configuraciones de poder más diferentes entre sí en términos cualitativos. La unipolaridad, por lo tanto, sería un estadio intermedio entre los sistemas de balance y los sistemas hegemónicos. Como vemos, el mundo unipolar de los últimos veinte años habría estado siempre más cerca de la multipolaridad, a modo de una uni-multipolaridad (Huntington 1993) que de la hegemonía.

5 Ambos son buenos ejemplos de una conducta particularmente trabajada por los neoclásicos: el «underbalancing» o liso y llano «nonbalancing», que pueden adoptar la forma de varios anglicismos: buck-passing, bandwagoning, appeasement, distancing o biding (Schweller 2004, 167) y permitieron en ambos casos el paso pacífico de un escenario bipolar a uno unipolar/hegemónico.
} 
decadencia gradual (Friedberg 1988). En esta sección no analizaremos ninguna de estas conductas en particular, sino las condiciones necesarias para que se produzcan o, dicho de otra forma, las condiciones necesarias para el cambio dentro del paradigma realista.

Tomaremos como punto de partida una sugerencia de Schweller $(2004,161)$ que intentaremos desarrollar: "Cuando el proceso decisorio y las relaciones estadosociedad más se asemejan al ideal de un actor unitario, las predicciones realistas son más acertadas. A la inversa, cuando los estados están divididos a nivel de la elite y de la sociedad, es menos probable que se comporten de acuerdo a las predicciones del balance de poder».

Es preciso notar que este principio general puede plantearse en términos racionalistas muy afines al neorrealismo: el cambio en la política internacional podría explicarse simplemente como producto de problemas de información que son, en esencia, problemas de comunicación de los incentivos sistémicos al Estado.

La socialización o isomorfismo —es decir, la interpretación de las estrategias exitosas en el mundo y su reproducción por parte de un estado en particulares uno de los supuestos esenciales del neorrealismo. Si este proceso no existiese, el Estado adoptaría medidas inconducentes, sería incapaz de obrar de acuerdo a la teoría del equilibrio de poder porque no conocería las capacidades de sus contrapartes ni tendría los conocimientos científicos, tecnológicos o técnicos para responder a los desafíos del sistema.

Si el actor no se está comportando racionalmente, entonces habrá que determinar cómo determinados actores internos receptan esta información internamente y la comunican al decisor, para luego analizar las fallas en ese proceso. En palabras de Gideon Rose $(1998,146)$ «las presiones sistémicas deben traducirse a través de variables intervinientes en el nivel doméstico». Pero ¿qué variables domésticas interfieren en la comunicación de estos incentivos? A nuestro entender son los grupos de interés y las burocracias en el interior del estado los que (i) acumulan las demandas de actores que perciben individualmente los incentivos sistémicos y (ii) las comunican a sus respectivos gobiernos a modo de demandas de políticas públicas, entre las cuales se encuentra la política exterior.

En este proceso, los canales de socialización son estos medios a través de los cuales la sociedad y el estado se comunican para que este último imite las conductas consideradas más eficientes en las distintas áreas de la vida social. Las formas que adquieren estos procesos de isomorfismo en el nivel microsocial exceden por mucho los límites de nuestra disciplina. Sin embargo, resulta de interés observar que la agregación de los individuos en grupos sociales organizados les permite coordinar las respuestas a estas presiones e introducir, siempre en el ámbito de sus competencias e intereses, demandas de políticas públicas al Estado con mayor eficacia (Olson 1965). Por lo tanto, tanto el grado de asociacionismo en la sociedad como la capacidad del estado aparecen como elementos centrales en la conformación de estos canales. 
Lo interesante aquí es que: si una sociedad dividida no recibe los incentivos sistémicos, no se organiza en grupos de interés, estos no son exitosos al transmitir sus demandas o el Estado es incapaz de receptarlas, la política exterior no puede ser racional en los términos expuestos por el neorrealismo. Las interferencias que se producen en estos complejísimos canales introducen en el mundo real la posibilidad de cambio.

Lo expuesto hasta aquí nos obliga a hablar de dos tipos ideales: (i) el «actor unificado» o sociabilizado y (ii) un "actor dividido».

El «actor unificado» es aquel que mantiene despejados los canales de socialización, de manera que los individuos pueden recibir sin interferencias los incentivos del exterior, asociarse y trasmitir sus demandas a un Estado receptivo, con agencias abiertas a esta información, capaces de analizarla y elaborar una respuesta consecuente. Nótese que si el mundo estuviese conformado únicamente por actores unificados no existirían los desbalances, tal como lo han planteado los neorrealistas utilizando juegos estratégicos con información perfecta (Grieco 1993) y por lo tanto no existiría el cambio.

La consideración anterior nos lleva a la tipología expuesta en el Cuadro 2.

Cuadro 2. Relación entre dos actores en virtud su sociabilidad.

\begin{tabular}{|c|c|c|c|}
\hline & \multicolumn{2}{|c|}{ B } \\
\hline & & Dividido & Unificado \\
\hline \multirow{2}{*}{$\mathbf{A}$} & $\frac{0}{\frac{0}{2}}$ & $\begin{array}{l}\text { No hay orden estable. Revisión del } \\
\text { sistema. }\end{array}$ & $\begin{array}{l}\text { Proceso de cambio. El actor B se } \\
\text { encuentra en una posición privilegiada } \\
\text { para dar forma al orden resultante. }\end{array}$ \\
\hline & 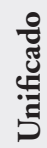 & $\begin{array}{l}\text { Proceso de cambio. El actor A se } \\
\text { encuentra en una posición privilegiada } \\
\text { para dar forma al orden resultante. }\end{array}$ & $\begin{array}{l}\text { Orden perfectamente estructurado. } \\
\text { No hay posibilidad de cambio. }\end{array}$ \\
\hline
\end{tabular}

En esta sección planteamos una teoría neoclásica del cambio que identifica como variable fundamental la capacidad de los grupos de interés para recibir y transmitir al Estado determinados incentivos sistémicos. Actores como los grupos de interés han aparecido muy tangencialmente en la literatura neoclásica (Ripsman 2009). La consideración de estos grupos nos ha permitido elaborar un planteo afín al neorrealismo, pues agregamos al modelo racionalista de Waltz una única variable cognitiva adicional: el grado de sociabilidad del Estado.

Hasta aquí solo hemos abordado una explicación a las condiciones que posibilitan el cambio dentro del paradigma neorrealista. Sin embargo, lo desarrollado en las secciones anteriores nos lleva a pensar que estas explicaciones 
son insuficientes a la hora de explicar cambios más radicales. En otras palabras, no creemos que exista únicamente una diferencia de cantidad —en este caso, de información- entre cambios ínfimos en las relaciones de poder y lo que hemos dado en llamar cambios de orden, aún cuando la existencia de actores más o menos «divididos» sea condición necesaria para que esto se produzca, este tipo de cambio implica una segunda modificación, en el carácter de dichos actores.

\section{Una explicación realista del cambio de orden: modelos de desarrollo y coaliciones sociales}

En esta sección postularemos que los cambios importantes en la política internacional - aquellos que modifican no solo el poder relativo, sino el orden jerárquico de los actores en el sistema—, no pueden explicarse simplemente a partir de problemas de información. Los cambios de mayor envergadura siempre han requerido una reestructuración de las sociedades mismas; que estas asuman un nuevo rol en el mundo, modificando las relaciones existentes entre sus mismos agentes domésticos y con el exterior.

Los grupos de interés no solo son importantes en tanto transmiten los incentivos sistémicos al estado, también son cualitativamente diferentes entre sí y cada uno de ellos introduce demandas específicas de acuerdo a los intereses de sus miembros. Por otro lado, los grupos de interés nunca están en igualdad de condiciones para obtener respuestas por parte del estado, pues únicamente algunos de estos grupos conforman una coalición social en la que el gobierno y, más generalmente, un determinado modelo de desarrollo, se sustentan. En otras palabras, incluir a los grupos de interés en el planteo neoclásico implicaría abrirnos en cierto grado a un análisis de economía política internacional.

Lo antedicho nos conduce a pensar que existen dos tipos de sesgo a la socialización: uno introducido por la capacidad de los grupos de interés de receptar y transmitir demandas al estado y otro debido a la preeminencia de determinados grupos de interés en detrimento de otros. Así, una unidad racional y eficiente no solo debería ser unitaria en el procesamiento de la información proveniente del sistema, sino también contar con la coalición adecuada de grupos de interés alineada con el gobierno. Como veremos, cuáles son las coaliciones más eficientes también es algo pasible de ser aprendido e imitado. Lo que pretendemos remarcar en este punto es que determinados grupos de interés y tipos de estado son preeminentes en los modelos de desarrollo de los actores más competitivos del sistema.

La idea de que distintas coaliciones sociales predominan en los distintos estados influyendo en su condición relativa de poder, de manera que determinados modelos económicos son característicos de los países dominantes y otros de los dominados, ha sido uno de los ejes principales de la teoría del sistema mundo 
(Wallerstein 1974), autores marxistas en general (Cox 1981; Strange 1988) y parte del pensamiento latinoamericano sobre política internacional (Puig 1980; Jaguaribe 1985). Esto no implica una ruptura tajante con la tradición realista que seguimos hasta el momento, sino una complementación necesaria. ${ }^{6}$

Nos figuramos a los efectos de una primera aproximación dos modelos de desarrollo predominantes. Es de desatacar que uno de estos tipos ideales será siempre característico de las grandes potencias, mientras que el modelo alternativo corresponderá a los estados subordinados. Llamaremos a estos tipos ideales (i) modelo central y (ii) modelo periférico.

La coalición del modelo central esta conformada por los grupos de interés que en un momento determinado de la historia demuestran hacer, en conjunto, eficiente y competitivo a un país. Esta coalición, por lo tanto, es estudiada y replicada por el resto de los actores. El modelo paradigmático, generalmente el de una gran potencia, incluye diferentes grupos de interés dependiendo del tiempo y el espacio en que se encuentre, pero esta coalición siempre existe.

En nuestros días podríamos identificar una coalición central más o menos similar en la cual el capital nacional —especialmente el industrial y comercial — y las fuerzas armadas son actores relevantes y hay una cierta inclusión de los sectores populares al modelo de desarrollo. En estos estados la burocracia es activa en la promoción de los intereses de la burguesía nacional allí donde se ve amenazada por el capital extranjero, promoviendo asimismo su expansión a otros mercados. Por otro lado, la coalición conformada por el capital extranjero, sectores agroexportadores, los servicios y las finanzas - pero con ausencia de la industria- y donde el estado asume un rol acotado en la defensa de los intereses nacionales en el ámbito económico, político y militar, parece ser la coalición característica de los modelos de (sub)desarrollo periféricos.

En el Cuadro 3 podemos apreciar una tipología que ilustra la interacción - siempre en un mundo de dos actores - entre actores centrales y periféricos. Como vemos, el tipo ideal de relación hegemónica y de balance de poder pueden explicarse razonablemente a partir de estas variables. En suma, en la integración del realismo de la periferia, el realismo neoclásico podría encontrar las herramientas para la integración de las distintas corrientes revisitadas en un programa realista unificado, sistematizado y coherente. Llegados a este punto podríamos concebir los distintos tipos de cambio o momentos teorizados por el realismo de la siguiente forma:

6 Esto último ubicaría nuestro planteo en los que Arlene Tickner (2002) llamó el «híbrido latinoamericano». En general estas teorías combinan preceptos del realismo clásico con ideas de la teoría de la dependencia o el "autonomistas» y han sido características del pensamiento sudamericano sobre esta materia. Agregamos que este eclecticismo también puede encontrarse en autores realistas clásicos como E. H. Carr (1964, 120-125) sin mucha dificultad y que el «eclecticismo analítico» es el método ideal para una disciplina social en reformulación como son las Relaciones Internacionales (Sil y Katzenstein 2005), tornándose más útil aún a la hora de analizar escenarios regionales concretos. 
(i) Cambios en el poder relativo. Para que el cambio se produzca es necesario que uno o más actores en el sistema posean un grado de socialización diferente, lo que posibilita un cambio en favor de los actores más cercanos al ideal «unitario» y en detrimento de los más cercanos al ideal «dividido».

(ii) Cambios en el orden relativo. Los cambios prolongados pueden terminar en una redefinición del orden de los actores. Países que participaban como grandes potencias en un equilibrio pueden verse relegados cambio estructural—, potencias hegemónicas pueden ser sucedidas por un competidor - cambio sistémico- o puede producirse un cambio de orden. En cualquiera de estos casos debe producirse un cambio en el carácter de la coalición social detrás de los estados en cuestión.

Cuadro 3. Relación entre dos actores en virtud de sus modelos de desarrollo

\begin{tabular}{|c|c|c|c|}
\hline & \multicolumn{2}{|c|}{ B } \\
\hline & & Central & Periférico \\
\hline \multirow{2}{*}{$\mathbf{A}$} & Uัँ & $\begin{array}{l}\text { Escenario de balance de poder. Los } \\
\text { actores compiten en su modelo de } \\
\text { desarrollo, no encuentran compatible. }\end{array}$ & $\begin{array}{l}\text { Escenario jerárquico. El actor } \mathrm{A} \text { es el } \\
\text { actor hegemónico y } \mathrm{B} \text { se subordina. }\end{array}$ \\
\hline & : & $\begin{array}{l}\text { Escenario jerárquico. El actor B se } \\
\text { consolida como actor hegemónico y A } \\
\text { se subordina. }\end{array}$ & $\begin{array}{l}\text { Ambos actores aceptan la hegemonía de } \\
\text { un tercer actor. Entre sí pueden tener } \\
\text { una relación jerárquica o competitiva. }\end{array}$ \\
\hline
\end{tabular}

El proceso de cambio de un equilibrio a una situación de hegemonía debería seguir la siguiente secuencia: i) uno de los actores —el más «dividido»— queda progresivamente rezagado provocándose tensiones que ii) como producto de una guerra o una crisis se solucionan con el reacomodamiento de sus grupos de interés internos a modo de un modelo "periférico" y subordinado en el marco de un nuevo sistema. En la siguiente sección veremos cómo un cambio de este estilo podría explicar i) el despegue del equilibrio y ii) la elevación hacia la hegemonía de Brasil en América del Sur.

\section{El ascenso de Brasil como potencia emergente desde una teoría realista integrada (1990-2010)}

Según lo que hemos planteado hasta el momento, el ascenso de Brasil supone que esta potencia ha logrado mantener aceitados sus canales de socialización, adoptado un modelo de desarrollo nacionalista/desarrollista — central - y una 
política exterior competitiva. El declive relativo de la Argentina frente a su competidor regional, en cambio, se debería a la preeminencia de un modelo de desarrollo internacionalista/neoliberal — periférico — en conjunción con un retraimiento del Estado que habría lesionado gravemente la capacidad de receptar las demandas de la sociedad y lo habría convertido en un estado más cercano al ideal «dividido». Si esto fuese así viviríamos en un mundo multipolar en el nivel sistémico y hegemónico en el nivel subsistémico o regional. Como sabemos, la realidad no responde perfectamente a estas características, pero aún así sostendremos que el fenómeno del ascenso de Brasil — y el descenso argentino — se debe en gran medida a que, en el largo plazo, estos estados estuvieron respectivamente más cerca de esos tipos ideales.

Interpretar a la Argentina y Brasil como dos países que han transitado este camino del equilibrio a la hegemonía, podría parecer contraintuitivo en virtud de sus asimetrías territoriales, demográficas y económicas. Sin embargo, es preciso recordar que durante la mayor parte del siglo XX, académicos, políticos, militares y diplomáticos a ambos lados de la frontera percibieron la relación en los términos de un equilibrio. Más aún, los estudios sobre la región son consistentes en atribuir la no guerra sudamericana previa a las transiciones democráticas a las políticas de balance de poder (Holsti 1996) que presentaban en términos subsistémicos una lógica bipolar argentino-brasilera (Martin 2006). Aunque esta situación se ha modificado ostensiblemente desde fines de los años ochenta, los efectos de aquella competencia aún pueden verse en una diplomacia argentina convencida de que las relaciones con Brasil deben plantearse en los términos de una paridad y sin abandonar la lógica del balance (Russell y Tokatlián 2003).

Pero aún si aceptamos que la relación entre Argentina y Brasil transitó en los últimos veinte años el camino del equilibrio a alguna otra cosa en sentido hacia una hegemonía brasileña, nos encontramos con el problema de las muchas variables de orden sistémico y subsistémico — regional o subregional— que debemos controlar para aplicar nuestra teoría a un estudio de dos casos (Hurrell 2007). Por ese motivo, es preciso dejar en claro que no intentaremos aquí una contrastación empírica, sino una brevísima ilustración histórico-comparada de las intuiciones teóricas presentadas.

En lo que respecta a la capacidad de socialización de los estados de Sudamérica en general, las reformas de los años noventa tuvieron dos efectos contradictorios. Por un lado desbloquearon muchos canales a través de los cuales se comunicaban los incentivos del mercado internacional a diferentes sectores de la economía por medio de la reducción arancelaria y otras políticas aperturistas. Por otro lado, contribuyó a un debilitamiento de las corporaciones, a un gran retraimiento del Estado y una disminución de su capacidad para receptar demandas de la sociedad y traducirlas en políticas públicas. 
Sin embargo, este proceso fue diferente en cada caso. Mientras la reforma del estado produjo un retraimiento notable del sector público en Argentina, hay sobradas razones para creer que los canales de comunicación con los principales grupos de interés se mantuvieron mucho más abiertos y fluidos en el caso brasileño —-facilitando su despegue —. La Constitución de 1988 permitió en Brasil una mayor proyección de las entidades empresariales otorgando un rol más activo al Congreso ${ }^{7}$ como receptor de estas demandas. Además, la reforma de la administración pública brasileña no obturó los vínculos del Ejecutivo con estos grupos de interés, creando nuevas agencias en reemplazo de las que debían cerrarse ${ }^{8}$.

Superados los tiempos álgidos de la reforma, la participación de la burocracia y los grupos de interés brasileños en la formulación de política exterior fue creciente. Los estudios sobre política exterior pasaron a conforman un campo disciplinar sorprendentemente desarrollado en Brasil - lo que es evidente en congresos, publicaciones, programas de posgrado, etc. - reflejando la complejidad de estos procesos y los muchos intereses involucrados.

En el caso argentino, aunque existieron intentos por sistematizar los estudios de política exterior (Russell 1990), hace décadas que no existe un debate al respecto. Esto no nos permite sacar conclusiones muy fehacientes sobre el carácter de los canales que vinculan a la sociedad con la política exterior, aunque es posible que la pérdida de importancia relativa de este campo disciplinar responda justamente a la desarticulación de los vínculos entre estado y sociedad en los años noventa y a la retracción de los grandes actores que influyeron históricamente en la política exterior como las fuerzas armadas y la burguesía nacional. A nuestro entender, esto

7 Justamente en el contexto de las reformas el poder legislativo adquirió importancia en la representación del sector empresario, fundamentalmente a través de prácticas de lobby y la representación empresaria en el Congresso Nacional. El lobbismo en Brasil se fue haciendo presente en trabajos de comisión y audiencias públicas, ya fuera a partir de una representación individual de la empresa por medio de consultores o a través de una representación colectiva del sector. La utilización de estas estrategias fue notoria en coyunturas críticas como la elaboración (1988) y revisión (1993, 1995-1996) de la Constitución, el debate de la Lei da Modernizacão dos Portos, y la Lei de Patentes. El debate sobre la cuestión del monopolio del petróleo, que incluyó el lobby de empresas extranjeras, grupos de presión nacionales como la Federação Única dos Petroleros (FUP) y la FIESP, y la apelación a manifestaciones públicas, es una muestra clara de las dimensiones que esta práctica adquirió en dicho contexto (Diniz y Boschi 2000, 70). Maria Regina Soares de Lima y Fabiano Santos (1998) aseguran que por los intereses redistributivos en juego, la cercanía de los legisladores con los grupos de interés entre su electorado y la mayor estabilidad que asegura su actuación como agente de veto, el ámbito del Congreso es ideal para la toma de decisión en materia de política exterior y comercial. El debate sobre el peso relativo del Congreso brasileño en esta materia esta abierto, aunque la evidencia sugiere que es cada vez más activo e influyente, a diferencia de lo que sucede con el Congreso argentino (Schenoni y Ferrandi Aztiria 2011).

8 Aunque hubo una reducción de las agencias donde los grupos de interés formulaban sus demandas al Estado durante el período militar, algunas instituciones como las câmaras setoriais —creadas en 1991- fueron exitosas en concertar los intereses estatales con los empresarios y laborales. Según Diniz y Boschi $(2000,63)$ las câmaras setoriais «tuvieron bastante éxito en el caso del sector automotor, en el que los acuerdos posibilitaron un ajuste creativo de cara a la apertura comercial, permitiendo al mismo tiempo el resurgimiento y mejoramiento del desempeño del complejo automotor en su conjunto». Instituciones luego impulsadas por Cardoso — y fortalecidas por Lulacomo la Câmara de Comércio Exterior (CAMEX) indican que la comunicación entre un empresariado competitivo y una burocracia especializada es un eje de la política exterior del Brasil emergente (Cason y Power 2009, 121). 
ha llevado a la concentración de la política exterior en unos pocos actores dentro del Poder Ejecutivo que explica el underbalancing frente a Brasil. ${ }^{9}$

En lo que respecta a los modelos de desarrollo y a una modificación económica más estructural del espacio regional, la elevación de Brasil hacia la posición de hegemón no es tan clara. ${ }^{10}$ Sin embargo, sabemos que las élites políticas y económicas brasileras, a diferencia de las argentinas, lograron mantener los ejes de un proyecto desarrollista a pesar del contexto neoliberal de los años noventa. En los términos de nuestro planteo teórico, Brasil solo habría liberalizado su economía en la medida en que esto permitió a sus empresarios una lectura más clara de los incentivos sistémicos tras décadas de proteccionismo exacerbado. Ante esa necesidad, el mismo Mercosur habría representado para Brasil una forma de moderar los efectos de la apertura multilateral, de modo que el país no degradara su perfil productivo ${ }^{11}$ ni se afectara la composición y organización del empresariado nacional. Cuando las medidas propuestas por el Ejecutivo amenazaron a los beneficiarios de políticas nacionalistas/desarrollistas, el Presidente encontró trabas de todo tipo a su accionar. El caso paradigmático es el de Collor, cuyos primeros intentos reformistas lo enfrentaron a grandes sectores de la burguesía nacional y otros factores de poder; la situación terminó por provocarle un notorio aislamiento político, la fractura de su coalición en el Congreso y un impeachment tras varios escándalos de corrupción.

La apertura de Brasil, además de limitada, fue mucho más gradual que la de Argentina, de manera que la coalición de origen desarrollista pudiera evaluar su sensibilidad y vulnerabilidad al cambio. Quizás por estas mismas razones el período neoliberal brasileño fue comparativamente muy breve —únicamente la presidencia de Collor y el primer período de Cardoso, podrían tenerse como aperturistas, excluyendo el interregno de Franco- de modo que Brasil fue uno

9 Es posible que la concentración de poder en el Ejecutivo argentino no sea privativa de la dinámica de la política exterior. En términos generales puede decirse del presidencialismo argentino que «los recursos institucionales y organizacionales disponibles facilitan la concentración de poder en el Ejecutivo, la delegación de poderes por parte del Congreso y la centralización de la toma de decisiones a través de los medios de producción de la disciplina partidaria, todo lo cual permite reducir el número de puntos de veto e incrementar, por ende, la posibilidad de que el Ejecutivo imponga cambios de política» (Bonvecchi 2002, 117). Sabemos que aunque algunos autores ven una situación similar en Brasil, el debate es notablemente más complejo (Palermo 2000). Sin embargo, si tratamos a la política exterior en particular, resulta evidente que, aún cuando la diplomacia presidencial parece un estilo cada vez más consolidado en Brasil —en detrimento de Itamaraty—, también es creciente la cantidad de actores institucionales y sociales que participan del proceso decisorio (Cason y Power 2009).

10 Una hegemonía en sentido estricto implicaría el control brasileño de acceso a materias primas y fuentes de capital una regulación propia de los mercados y la posesión de ventajas competitivas en la producción de los bienes de mayor valor agregado (Keohane 1984,32). Claramente este concepto no refleja la realidad de la relación bilateral ni del sistema sudamericano.

11 Un análisis de la composición de las exportaciones brasileñas durante los años noventa demuestra que estas estuvieron conformadas en su mayoría por bienes manufacturados, mientras que las importaciones presentan una participación relativamente baja de bienes de consumo (Petrecolla 2004, 39), Argentina, en cambio, primarizó su perfil productivo y exportador. 
de los últimos países en adoptar las reformas y uno de los primeros en abandonar el modelo neoliberal con la devaluación del real en 1999.

En la Argentina, las políticas neoliberales habían comenzado antes y se mantendrían incluso en un contexto recesivo hasta estallar en una de las peores crisis económicas de la historia del país. Por lo demás, los gobiernos reformistas se encargaron de aplicar una reforma profunda, dividiendo y desarticulando en lo posible todas las corporaciones que participaban de la coalición mercado-internista (Etchemendy 2001) y modificando notablemente el perfil productivo del país.

El modelo resultante del colapso del 2001, aun tras el cambio de paradigma que sobrevino con las administraciones de Eduardo Duhalde, Néstor Kirchner y Cristina Fernández, no se asemeja a lo que hemos dado en llamar un modelo nacionalista/desarrollista mucho más allá de lo discursivo (Grugel y Riggirozzi 2007), ya sea por la imposibilidad de adquirir un perfil productivo de mayor valor agregado, o por la exclusión de sectores del empresariado nacional y otros actores centrales de una coalición "central» como son las fuerzas armadas. ${ }^{12}$

En síntesis, Brasil habría logrado mantener un modelo nacionalista/ desarrollista mientras que la Argentina, a pesar del perfil de política económica adoptado tras la crisis del paradigma neoliberal, pareciera no poder conformar una coalición estable de este tipo. Es interesante notar que aquel período en que ambos países coincidieron en un modelo más o menos neoliberal y una relación más estrecha con los Estados Unidos fue el período más cooperativo, que cuenta en su haber al Mercosur como máxima expresión de esa cooperación. Más tarde, el viraje a un modelo exitosamente neodesarrollista en Brasil produjo crecientes tensiones. Allí pareciera encontrarse el origen del largo letargo que vive el proceso de integración. Las indefiniciones en el modelo de desarrollo y la política exterior argentina, así como los esfuerzos de Itamaraty por no perder un mínimo liderazgo regional necesario para su proyección global (Soares de Lima 2007), habrían permitido ocultar este problema bajo un manto de confluencia ideológica. Así y todo, la divergencia de intereses entre Brasil y el resto de la región es cada vez más evidente (Malamud 2011).

\section{Conclusiones}

Este trabajo contribuye a una comprensión de las potencias emergentes integrando dos corrientes neorrealistas: la «teoría del equilibrio de poder» y la «teoría de la estabilidad hegemónica». En este esfuerzo teórico proponemos un camino para generar en el programa realista un enfoque útil para el análisis del cambio en

12 La cuestión de los militares en Argentina es a todas luces un punto relevante. Como producto de las violaciones a los Derechos Humanos, los fracasos económicos, la derrota de Malvinas y algunos levantamientos en democracia, los militares argentinos sufrieron una reducción notable de su presupuesto, el desmantelamiento de la mayoría de sus proyectos y perdieron la legitimidad necesaria para articular y hacer oír sus demandas. 
la política internacional. Asimismo, la convergencia de ideas desarrollistas con el realismo neoclásico podría generar una variante normativamente más progresista del realismo, opción que parecen haber preferido históricamente los intelectuales sudamericanos.

En lo que respecta al estudio de América del Sur este modelo podría explicar al menos dos preguntas importantes ¿qué características de la política exterior del Brasil han logrado constituirlo en una potencia emergente? ¿YY por qué razón la Argentina o el resto de la región no han problematizado suficientemente el ascenso y la eventual hegemonía de su principal contraparte regional?

En respuesta a la primera pregunta podemos decir que Brasil preservó su capacidad estatal al frente de un proyecto desarrollista basado en una coalición social estable. En otras palabras, Brasil parece haberse regido por un ideal de política exterior «autonomista», preservando su esfera de acción con el objetivo de abandonar una posición económica y política subordinada en el sistema internacional a partir de la emulación de un modelo de desarrollo central. La respuesta a la segunda pregunta es simple: la Argentina y la región han tomado el camino inverso.

La consecuencia lógica de este proceso es que Brasil sea percibido como una potencia emergente y la Argentina, desorientada e indefinida, deba optar eventualmente por un modelo que se acople al brasileño o competir. Claro está, la diplomacia brasileña podría afectar el resultado de este proceso tornando más o menos inclusivo su liderazgo del bloque, pero los costos de la hegemonía son más caros en tiempos de crisis.

\section{Referencias bibliográficas}

BERLINSKI, Julio (2004) Los impactos de la politica comercial: Argentina y Brasil (1988-1997), Instituto Di Tella, Siglo XXI, Buenos Aires.

BONVECCHI, Alejandro (2002) «Estrategia de supervivencia y tácticas de disuasión», en NOVARO, M. (comp.) El derrumbe politico en el ocaso de la convertibilidad, Norma, Buenos Aires, p. 109-192.

CASON, Jeffrey \& POWER, Timothy (2009), «Presidentialization, Pluralization, and the Rollback of Itamaraty: Explaining Change in Brazilian Foreign Policy Making in the CardosoLula Era», en International Political Science Review, Vol. 30, No. 2, p. 117-40.

COX, Robert (1981) «Social forces, states and world orders: beyond international relations theory», en Millennium: Journal of International Studies, Vol. 10, No. 2, p. 126-155.

DINIZ, Eli \& BOSCHI, Renato (2000) «Globalização, herança corporativa e a representação dos interesses empresariais: novas configurações no cenário pós-reformas», en BOSCHI, R. et al. Elites políticas e econômicas no Brasil contemporâneo: a desconstrução da ordem corporativa e o papel do Legislativo no cenário pós-reformas, São Paulo, Fundação Konrad Adenauer, p. 15-87.

ETCHEMENDY, Sebastián (2001) «Construir coaliciones reformistas: la política de las compensaciones en el camino argentino hacia la liberalización económica», en Desarrollo Económico, vol. 40, n. ${ }^{\circ}$ 160, p. 675-706. 
FERREIRA VIDIGAL, Armando (1999) «Las Fuerzas Armadas y la Política de Defensa en el Brasil y la Argentina» en LLADÓS, J. M. \& PINHEIRO, S. Perspectivas: Brasil y Argentina, IPRI/CARI, Buenos Aires, p. 75-96.

FRIEDBERG, Aaron (1988) The Weary Titan: Britain and the Experience of Relative Decline, 1895-1905, Princeton University Press, Princeton.

GILPIN, Robert (1981) War and Change in International Politics, Cambridge, Cambridge University Press.

GRIECO, Joseph (1993) «Understanding the Problem of International Cooperation: The Limits of Neoliberal Institutionalism and the Future of Realist Theory», en BALDWIN, D. (ed.) Neorealism and Neoliberalism: The Contemporary Debate, New York, Columbia University Press.

GRUGEL, Jean \& RIGGIROZZI, Pia (2007) "The return of the state in Argentina», en International Affairs, Vol. 83, No. 1, p. 87-107.

HANDEL, Michael (1981) Weak States in the International System, London, Frank Cass \& Co. HOLSTI, Kalevi (1996) The State, War, and the State of War, Cambridge University Press.

HUNTINGTON, Samuel (1993) «Why International Primacy Matters» en International Security, Vol. 17, No. 4, p. 68-83.

HURRELL, Andrew (2007) «One world? Many worlds? The place of regions in the study of international society», en International Affairs, Vol. 83, No. 3, p. 127-143.

JAGUARIBE, Hélio (1985) El nuevo escenario internacional, Autonomía periférica y hegemonía del centro, Fondo de Cultura Económica, México.

KEOHANE, Robert (1984) After Hegemony, Princeton, Princeton University Press.

LOBELL, Steven et al. (2009) Neoclassical Realism, the State and Foreign Policy, Cambridge, Cambridge University Press.

MALAMUD, Andrés (2011) «A Leader without Followers? The Growing Divergence Between the Regional and Global Performance of Brazilian Foreign Policy», en Latin American Politics and Society, Vol. 53, No. 3, p. 1-24.

MARTIN, Félix (2006) Militarist Peace in South America: Conditions for War and Peace, New York, Palgrave.

MEARSHEIMER, John (2001) The Tragedy of Great Power Politics, New York, Norton.

OLSON, Mancur (1965), The Logic of Collective Action, Cambridge, Harvard University Press.

PALERMO, Vicente (2000) «¿Cómo se gobierna Brasil? El debate brasileño sobre instituciones políticas y gestión de gobierno», en Desarrollo Económico, Vol. 40, n. ${ }^{\circ}$ 159, p. 493-517.

PANG, Eul-Soo (2002) The International Political Economy of Transformation in Argentina, Brazil and Chile since 1960. Palgrave Macmillan, New York.

PUIG, Juan Carlos (1980) Doctrinas internacionales y autonomía latinoamericana, Instituto de Altos Estudios de América Latina, Caracas.

RIPSMAN, Norrin (2009) «Neoclassical realism and domestic interest groups», en ROSE, Gideon (1998) "Neoclassical Realism and Theories of Foreign Policy», en World Politics, Vol. $51,{ }^{\circ}$ 1, p. $144-172$. 
RUSSELL, Roberto (1990) «El proceso de toma de decisiones en la política exterior argentina», en RUSSELL, R. (ed.) Política Exterior y toma de decisiones en América Latina, Grupo Editor Latinoamericano, Buenos Aires.

RUSSELL, Roberto \& TOKATLIAN, Juan Grabriel (2003) El lugar de Brasil en la política exterior argentina, Buenos Aires, Fondo de Cultura Económica.

RUSSELL, Roberto \& TOKATLIAN, Juan Gabriel (2010) Autonomía y neutralidad en la globalización, Capital Intelectual, Buenos Aires.

SCHENONI, Luis \& FERRANDI AZTIRIA, Alejo (2011) «El Congreso en la formulación de política exterior: Argentina y Brasil en perspectiva comparada», en Revista Densidades, n. 7.

SCHWELLER, Randall (1996) «Neorealism's Status Quo Bias: What Security Dilemma?», en Security Studies, Vol. 5, No. 3, p. 90-121.

SCHWELLER, Randall (1998) Deadly Imbalances: Tripolarity and Hitler's Strategy of World Conquest.

SCHWELLER, Randall (2004) «Unanswered Threats: A Neoclassical Realist Theory of Underbalancing», en International Security, Vol. 29, No. 2, p. 159-201.

SIL, Rudra \& KATZENSTEIN, Peter (2005) «What is Analytic Eclecticism and Why Do We Need It? A Pragmatic Perspective on Problems and Mechanisms in the Study of World Politics», trabajo presentado en: Annual meeting of the American Political Science Association, Washington DC. Disponible (18/05/2011) en: http://www.allacademic.com/meta/p41957_index.html

SNYDER, Jack (1991) Myths of Empire: Domestic Politics and International Ambition, Cornell University Press, New York.

SOARES DE LIMA, Maria Regina (2007) «Brasil como país intermedio: imprecisión conceptual y dilemas políticos», en TOKATLIAN J. G. (comp.) India, Brasily Sudáfrica. El impacto de las nuevas potencias regionales, Ed. del Zorzal, Buenos Aires.

SOARES DE LIMA, Maria Regina \& SANTOS, Fabiano (1998) «Brazilian Congress and Foreign Trade Policy", presentación en el congreso de la Latin American Studies Association, 24 al 26 de septiembre, Chicago.

STRANGE, Susan (1988) States and Markets, London, Pinter.

TICKNER, Alvine (2002) Los estudios internacionales en América Latina. ¿Subordinación intelectual o pensamiento emancipatorio?, Bogotá, Alfaomega.

WALLERSTEIN, Immanuel (1974) «The Rise and Future Demise of the World Capitalist System: Concepts for Comparative Analysis», Comparative Studies in Society and History, Vol. 16, No. 4, p. 387-415.

WALT, Stephen (2002) "The Enduring Relevance of the Realist Tradition», en en KATZNELSON, I. y MILNER, H. (eds.) Political Science: The State of the Discipline III, New Tork, Norton, p. 202.

WALTZ, Kenneth (1993) «The Emerging Structure of International Politics», en International Security, Vol. 18, No. 2, p. 44-79.

WILKINSON, David (1999) «Unipolarity without Hegemony», en International Studies Review, Vol. 1, No. 2, p. 141-172.

WRIGHT, Martin (1979) Power Politics, Holmes and Meir, New York. 
WOHLFORTH, William (1993) The Elusive Balance: Power and Perceptions during the Cold War, Ithaca.

WOHLFORTH, William (1995) «Realism and the End of the Cold War», en International Security, Vol. 19, No. 3, p. 91-129.

ZAKARIA, Fareed (2000) De la riqueza al poder. Los origenes del liderazgo mundial de Estados Unidos, GEDISA, Barcelona.

ZAKARIA, Fareed (2008) The Post-American World, Allen Lane, New York.

Recibido el 9 de agosto de 2011 Aceptado el 6 de octubre de 2011

\section{Resumen}

El presente trabajo se enmarca en el actual debate sobre potencias emergentes intentando un aporte desde una perspectiva latinoamericana. Para ello trabajará algunas disyuntivas teóricas que se han tornado difíciles de resolver para el mainstream racionalista de la disciplina, desarrollando un modelo de cambio complementario del programa realista actual. Argumentaremos que este marco presenta algunas ventajas para el estudio de diversas cuestiones relativas a las relaciones de poder en América del Sur, destacando en particular dos preguntas: ¿qué características de la política exterior del Brasil han logrado constituirlo en una potencia emergente? ¿Y por qué los países de la región no han desarrollado una política exterior acorde al desafío que significa el ascenso de su principal contraparte regional?

Palabras clave: Brasil; política exterior; potencias emergentes; relaciones internacionales; Sudamérica.

\section{Abstract}

This article is framed within the current debate on emerging powers from a Latin American perspective. It tries to solve some of the theoretical problems of the mainstream rationalist approach in IR theory, proposing a model of change rooted in the current realist program. This framework is applied to the study of power politics in South America with the goal of answering two major questions: why has Brazilian foreign policy been so successful in positioning this country as an emerging power? And why have not any country in the region reacted accordingly to the challenge posed by its mayor regional counterpart rise?

Keywords: Brazil; foreign policy; emerging powers; International Relations; South America. 